\title{
Patients with traumatic brain injury relayed neutral or positive self narratives for coping with their changed lives
}

Nochi M. Reconstructing self-narratives in coping with traumatic brain injury. Soc Sci Med 2000 Dec;51:1 795-804.

\author{
QUESTION: How do patients with traumatic brain injury (TBI) cope with their changed \\ lives?
}

\section{Design}

Qualitative study using open ended interviewing and participant observation.

\section{Setting}

TBI support group in a city in the eastern US.

\section{Patients}

10 adult patients (mean age 38 y, range $27-54$ y, 80\% men) with TBI (mean time since injury 9.5 y) who lived in the community after hospital discharge, had the ability to participate in in-depth interviews, and were interested in discussing their experiences.

\section{Methods}

The investigator conversed with the patients at an independent living centre or participated in their daily living activities to build a rapport and then had $\geqslant 2$ semistructured interviews lasting 45-60 minutes with each patient. Patients were asked open ended questions about their background, rehabilitation activities, present situations, and future plans. Patients' thoughts and feelings about themselves were elicited. The grounded theory method was used to group the emerging remarks and actions into concepts that were compared across patients' data sets.

\section{Main findings}

5 types of self narratives for coping with TBI emerged that were mentioned by $\geqslant 3$ patients. The narratives were aimed at obtaining a neutral or positive self image. The self better than others was mentioned by 7 patients who contrasted their present situation with ones that could be worse. The grown self was mentioned by 6 patients who felt that certain positive outcomes could be attributed to their TBI and that they had grown as individuals or acquired new insight into themselves or other people. The recovering self was mentioned by 4 patients who saw themselves as progressing towards the level they had before their TBI. The self living here and now was mentioned by 4 patients who maintained a feeling of self worth without feeling compelled to compare themselves with other people or their pre-injury selves. Narratives included such statements as "Normal is what you believe. I believe I'm normal," "I live for today... and I don't worry about tomorrow," and "one day at a time." The protesting self was mentioned by 3 patients who discussed how best to function in society with a TBI or with disability in general.

\section{Conclusions}

5 common types of self narratives emerged among 10 people with traumatic brain injury for coping with their changed lives. The narratives aimed at obtaining a neutral or positive self image.

\section{COMMENTARY}

In this analysis of feedback from patients with TBI, Nochi explores how those with relatively positive responses to their injuries construct "personal narratives". His work provides a richly textured qualitative discussion of the means used by patients to adapt and adjust to the realities of life after cerebral trauma. This type of naturalistic anthropological research into TBI is difficult to do, but the insights it offers can be of great importance to clinicians and caretakers. Nochi is correct in warning us from the outset that his work is without broad generalisability. His sample is small, even by qualitative research standards. Only 10 patients were interviewed, and all were people who, by virtue of their verbal facility, insight, and current living situation, were among the least injured and most well adapted of those with TBI.

Nochi used extensive field notes, which he analysed for critical themes. He included his own reflections on each patient as "objective" data for analysis. From these, he noted elements that recurred most frequently and used data from early patients to sculpt concepts that guided the coding of later patients. Issues that recurred most frequently were noted in order of pervasiveness in the 10 patients.

I confess to 2 conflicting responses to this study. On the one hand, the themes Nochi identifies are fascinating, well conceptualised, and expand our notions of how those with substantial brain injuries reconstruct their sense of the world and themselves. He provides elegant views of the interplay of time, place, and role in these patients' changing self stories, providing a framework within which we can begin to organise our own patients' experiences. Thus, he offers a basis for initiating a process of learning and understanding how differing rehabilitative approaches might capitalise on these distinct self views to aid the process of maximising adaptation and functioning.

On the other hand, this study stretches the boundaries of what we mean by "evidence-based". Nochi seems to struggle at the boundaries of quantitative and qualitative analyses. He barely notes that, by using his own responses to the narratives as subject for thematic analysis, he may be shaping the data as much as any of his subjects. This is a non-representative sample, studied without objective analytic tools, and with no attempt to correlate patients' characteristics and personal themes: are the women more optimistic than the men? Do older patients differ from younger in their defences? With this study, are we reverting to case studies which, using the language of the new millennium, are now (re)presented as somehow more data driven than we'd previously supposed?

Stephen M Goldfinger, MD State University of New York/Downstate Brooklyn, New York, USA
For correspondence: Dr M Nochi,

Department of Psychology, Teikyo University, 359 Otsuka, Hachioji-shi, Tokyo 192-0395, Japan. Fax +81426 783439. 